ARTICLE

https://doi.org/10.1038/s41467-021-21659-6

\title{
Millimeter wave photonics with terahertz semiconductor lasers
}

Valentino Pistore ${ }^{1}$, Hanond Nong ${ }^{1}$, Pierre-Baptiste Vigneron², Katia Garrasi ${ }^{3}$, Sarah Houver (i) ${ }^{4}$, Lianhe Li (i) ${ }^{5}$, A. Giles Davies (1D ${ }^{5}$, Edmund H. Linfield (1D 5 ${ }^{5}$, Jerome Tignon ${ }^{1}$, Juliette Mangeney ${ }^{1}$, Raffaele Colombelli (i) ${ }^{2}$, Miriam S. Vitiello (i) ${ }^{3} \&$ Sukhdeep S. Dhillon (id ${ }^{1 \times}$

Millimeter wave (mmWave) generation using photonic techniques has so far been limited to the use of near-infrared lasers that are down-converted to the mmWave region. However, such methodologies do not currently benefit from a monolithic architecture and suffer from the quantum defect i.e. the difference in photon energies between the near-infrared and $\mathrm{mmW}$ ave region, which can ultimately limit the conversion efficiency. Miniaturized terahertz $(\mathrm{THz})$ quantum cascade lasers (QCLs) have inherent advantages in this respect: their low energy photons, ultrafast gain relaxation and high nonlinearities open up the possibility of innovatively integrating both laser action and mmWave generation in a single device. Here, we demonstrate intracavity mmWave generation within $\mathrm{THz}$ QCLs over the unprecedented range of $25 \mathrm{GHz}$ to $500 \mathrm{GHz}$. Through ultrafast time resolved techniques, we highlight the importance of modal phases and that the process is a result of a giant second-order nonlinearity combined with a phase matched process between the $\mathrm{THz}$ and mmWave emission. Importantly, this work opens up the possibility of compact, low noise mmWave generation using modelocked $\mathrm{THz}$ frequency combs.

\footnotetext{
${ }^{1}$ Laboratoire de Physique de l'Ecole Normale Supérieure, ENS, Université PSL, CNRS, Sorbonne Université, Université de Paris, Paris, France. ${ }^{2}$ Centre de Nanosciences et de Nanotechnologies (C2N), CNRS UMR 9001, Université Paris-Saclay, Palaiseau, France. ${ }^{3}$ NEST, CNR - Istituto Nanoscienze and Scuola Normale Superiore, Piazza San Silvestro 12, Pisa, Italy. ${ }^{4}$ DOTA, ONERA, Université Paris-Saclay, Palaiseau, France. ${ }^{5}$ School of Electronic and Electrical Engineering, University of Leeds, Leeds, UK. ${ }_{\text {email: sukhdeep.dhillon@ens.fr }}$
} 
T he ability to generate millimetre (mmWave) waves $(30-300 \mathrm{GHz})$ from highly stable lasers promises to be a key method to achieve the spectrally pure, low phase noise and microwave signals that are targeted for local oscillators in high frequency communications ${ }^{1-3}$. Examples have been shown by heterodyning two optical lasers ${ }^{4}$, opto-electronic oscillators ${ }^{5}$ and pulsed lasers ${ }^{6}$, in a nonlinear medium or a fast photodetector to generate microwaves signals. This field of microwave photonics has also been revolutionised by the advent of the optical frequency comb, where the beating of the highly stable modes can generate low noise and high purity microwave emission ${ }^{7-9}$. Most of these methods, however, use lasers operating in the visible or near-infra-red part of the electromagnetic spectrum, leading to orders of magnitude difference in photon energy between the generating laser photon energy and that of the microwave photon - the quantum defect. This inherently limits the efficiency of the system $\sim E_{\mathrm{GHz}} / E_{\mathrm{opt}}$, where $E_{\mathrm{opt}}$ is typically $1 \mathrm{eV}$ and $E_{\mathrm{GHz}}<1$ $\mathrm{meV}$. Finally, microwave photonic systems based on optical frequency combs are typically used in a non-integrated approach, where the optical laser is separated from the fast photo-mixer or nonlinear crystal $^{7,9,10}$. Although a range of electronic methods can be used to generate directly or through upconversion mmWave emission ${ }^{11-13}$, these sources typically suffer from high phase noise and therefore not as spectrally pure as photonic based sources $^{7,14}$. In this work we show that Terahertz (THz) frequency quantum cascade lasers (QCLs) ${ }^{15}$, with their inherently low photon energies, two orders of magnitude smaller than in the optical range, can be used as an integrated platform to generate free space emission over the entire mmWave region $(0.02-500$ $\mathrm{GHz}$ ) via intracavity nonlinear beating of the laser longitudinal modes.

$\mathrm{THz}$ QCLs operate between 1.2 and $5 \mathrm{THz}$, corresponding to photon energies of $5-21 \mathrm{meV}$, and possess ultrafast dynamics and giant nonlinearities ${ }^{16}$ permitting an integrated approach where the laser excitation and mmWave generation are realised within the same laser cavity. THz QCLs have achieved impressive performances in term of spectral coverage ${ }^{17}$, output power $^{18}$ and bandwidth ${ }^{19,20}$ via carefully designed waveguides and active regions. Further, temperature operation has been extended to Peltier cooled operation 21,22 , with recent results showing operation up to $250 \mathrm{~K}^{23}$ with further perspectives to achieve room temperature operation through new bandstructure designs and materials ${ }^{24}$. To extend the capabilities of these devices, nonlinear intracavity processes have been successfully exploited to generate frequencies in the telecom band $\sim 1.55 \mu \mathrm{m}^{25,26}$, and shown the generation of frequency combs $^{27-29}$ with octave spanning bandwidth ${ }^{20}$. Furthermore, difference-frequency generation (DFG) in mid-infrared QCLs has shown the possibility of efficient $\mathrm{THz}$ generation at room temperature ${ }^{30-33}$. These demonstrations illustrate the giant optical nonlinearities that can occur within QCLs (greater than $\sim 10^{5} \mathrm{pm} / \mathrm{V}$, compared to $10^{2} \mathrm{pm} / \mathrm{V}$ for GaAs) and how they can be used to access different spectral regions. However, the possibility of accessing the entire mmWave region, either through direct or nonlinear generation, has yet to be demonstrated. Recently, mid-infrared QCL-based frequency combs were shown to generate free space microwave radiation through beating of the comb lines in an integrated approach ${ }^{34,35}$. This takes advantage of the fast QCL dynamics ( 1 ps), suggesting potential operation even up to $\mathrm{THz}$ frequencies, and highlighted both electronic and photonic methods to generate electromagnetic radiation within QCLs. However, such microwave emission has, so far, been limited to low frequencies with optical beatnote detection up to $13 \mathrm{GHz}$ shown on a Schottky mixer $^{36}$. This results in a large spectral gap between 15 and 500 $\mathrm{GHz}$ for QCL-based microwave generation.
Here we demonstrate free space mmWave generation in a set of THz QCLs with different dimensions, emission bandwidth and spectral response. As a common characteristic, all devices show mmWave emission lines, equally spaced in frequency by the QCL round-trip frequency. Depending on the bandwidth of the $\mathrm{THz}$ QCL employed and the dispersion of the $\mathrm{THz}$ lines, we realise mmWave emission up to $500 \mathrm{GHz}$. Moreover, we demonstrate that the frequency of the mmWave lines can be engineered by designing a QCL with two-separated THz spectral bands, with the formation of the mmWave emission measured on ultrafast timescales. We also highlight the important role of modal phases for DFG and we show here that the mmWave generation is a result of a phase matched process, owing to low loss waveguides and to the phase of the mmWave emission being equal to the envelope of the THz emission. Finally, we show a giant secondorder nonlinearity from the QCL bandstructure, owing to the closely spaced sublevels in energy that directly permits a nonlinearity in the mmWave range. This could support the stabilisation of QCL-based frequency combs, in addition to four-wave mixing.

\section{Results}

To characterise the entire QCL emission from 10 to $4000 \mathrm{GHz}$, we employ an injection seeding technique based around electro-optic detection in a THz-TDS system. As well as enabling ultrafast time-resolved measurements, this proven technique provides a detection bandwidth much greater than spectrum analysers and limited only by the cut-off frequency of the $\mathrm{ZnTe}$ electro-optic detection crystal $^{37}$. To demonstrate mmWave generation, a coherent terahertz pulse generated from an ultrafast laser is injected into the QCL, and at the same time it is switched on with an ultrafast electrical pulse that is synchronised to the $\mathrm{THz}$ pulse. The pulse is amplified to the steady state where it seeds the QCL emission. This permits free space coherent detection of the QCL time-resolved electric field using a second beam from the same ultrafast laser ${ }^{38}$. The technique has shown to be equivalent to laser action on the inherent QCL spontaneous emission such that the measured time profile corresponds to the free-running case ${ }^{39}$. A squared Fourier transform operation is applied to the measured time-domain trace in order to extract the QCL intensity spectrum. In this study, three QCLs with lasing frequencies between 2 and $3 \mathrm{THz}$ with metal-metal waveguides ${ }^{40,41}$ were used. For $\mathrm{QCL}_{1}$ and $\mathrm{QCL}_{3}$, the active region design is based on a $2.4-\mathrm{THz}$ three well ${ }^{42,43}$ structure, whilst $\mathrm{QCL}_{2}$ is based on a $2.8-\mathrm{THz}$ heterogeneous stacked nine well design ${ }^{29,44,45}$ for broadband operation $(\sim 800 \mathrm{GHz})$. $\mathrm{QCL}_{1}$ shows laser action with a bandwidth of $\sim 500 \mathrm{GHz}$ and is $1.5 \mathrm{~mm}$ long and $60 \mu \mathrm{m}$ wide. $\mathrm{QCL}_{2}$ is $2.9 \mathrm{~mm}$ long and $85 \mu \mathrm{m}$ wide. Finally, $\mathrm{QCL}_{3}$ was designed for two lasing bands separated by $\sim 240 \mathrm{GHz}$ using an integrated GiresTournois interferometer ${ }^{46}$ (GTI) and is $3 \mathrm{~mm}$ long and $68 \mu \mathrm{m}$ wide. The GTI is $60 \mu \mathrm{m}$ long, and separated from the main QCL section by $4 \mu \mathrm{m}$. Each QCL is used to show a range of spectral responses in the mmWave generation as discussed below.

The emission properties of $\mathrm{QCL}_{1}$ are shown in Fig. 1. The temporal response (Fig. 1a) shows the typical profile from these devices ${ }^{38,43}$ with a strong amplitude modulation that can arise from the QCL ultrafast dynamics ${ }^{39}$. The corresponding frequency spectrum (Fig. 1b) shows emission centred at $2.42 \mathrm{THz}$ with Fabry-Pérot modes separated by the round-trip frequency $\left(f_{R T}\right)$. Owing to the broadband nature of the coherent detection, the spectrum also clearly shows spectral modes covering the mmWave region between $26 \mathrm{GHz}$ and $367 \mathrm{GHz}$, also separated by exactly $f_{R T}$. In order to rule out any spectral artefact originating from nonlinearities in the measurement arrangement that are not associated with the QCL, we additionally conducted the 


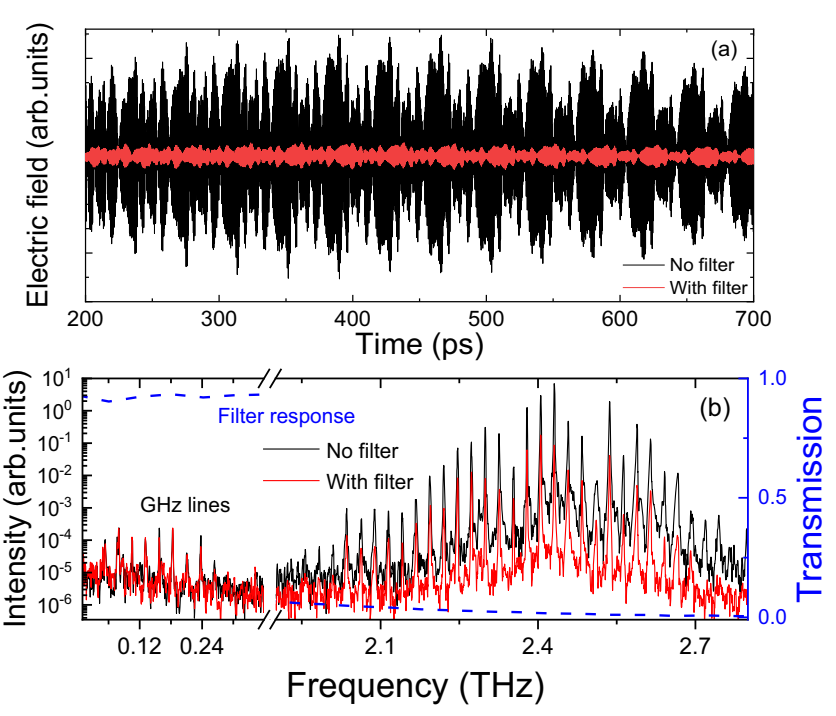

Fig. 1 Temporal and spectral response of $\mathbf{m m W a v e}$ generation from QCL1. a Time-resolved electric field from injection seeded QCL with (red) and without (black) the low-pass filter. Electric field oscillations of the laser emission are not visible owing to the long temporal scan. b Intensity spectrum of emission from $\mathrm{QCL}_{1}$ with (red) and without (black) the low pass filter. The QCL is driven in pulsed mode (10\% duty cycle) and at a fixed heat sink temperature of $10 \mathrm{~K}$. The $\mathrm{THz}$ emission is centred around 2.4 THz. mmWave generation is clearly observed. The filter (blue curve) removes an order of magnitude of the intensity of the $\mathrm{THz}$ emission but does not change the mmWave emission. This unambiguously illustrates the mmWave emission is from the QCL and not an artefact of the detection technique.

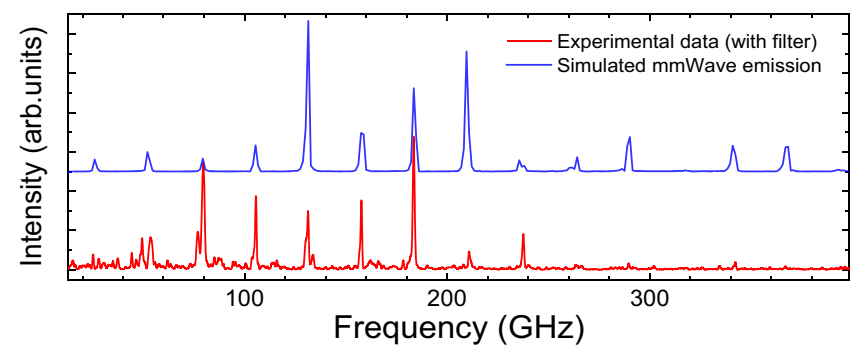

Fig. 2 Enhanced view of $\mathrm{mmW}$ ave spectral emission from $\mathrm{QCL}_{1}$ (red) showing comparison with simulated results (blue) from the QCL THz spectrum. mmWave emission is observed up to $367 \mathrm{GHz}$.

investigations employing a 500-GHz low-pass filter (Spectrasil B). The transmission spectrum is shown in Fig. 1b (blue dotted curve). Although the $\mathrm{THz}$ signal intensity is attenuated by more than one order of magnitude, the mmWave lines remain the same, demonstrating that the emission originates from the QCL. An expanded view of the mmWave spectrum is shown in Fig. 2. Two observations can be made. First, there is an equal mode spacing between the $\mathrm{THz}$ and the mmWave lines $(\sim 26 \mathrm{GHz})$, with the latter's bandwidth limited to the bandwidth of the $\mathrm{THz}$ emission. Second, the position and intensity of the mmWave lines can be evaluated by calculating the expected positions from the measured $\mathrm{THz}$ temporal emission whilst assuming a secondorder nonlinearity for DFG. This is done by calculating the Fourier transform of the square of the $\mathrm{THz}$ temporal emission (blue curve in Fig. 2), as the second-order polarisation, $P^{2 n d}$, is proportional to the square of the electric field, $E(t)$. This allows the phase of each mode to be automatically considered in the calculated emission spectrum. The effect of the phase is discussed further below in the case of $\mathrm{QCL}_{2}$. The collection and detection

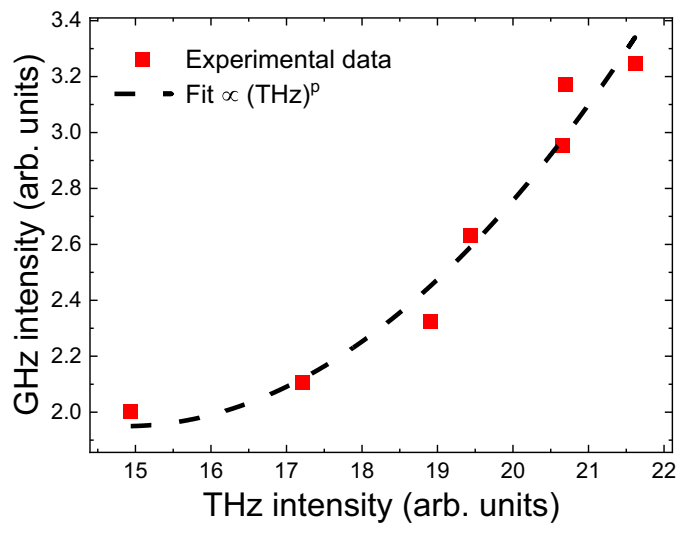

Fig. 3 Observation of second-order nonlinearity. mmWave intensity as a function of $\mathrm{THz}$ intensity (red squares) illustrating a nonlinear behaviour with an exponent of $P=2.1$ (fit, black dashed line) corresponding to a second-order susceptibility. The measured intensities correspond to the integrated emission in each spectral band.

efficiencies are included in the calculation and were estimated by 3D simulations of the far-field (COMSOL Multiphysics) and the waist size of each frequency at the detection crystal, respectively. These factors lead to reduced detection response to low-frequency mmWave emission and further details are highlighted in the supplementary material Figs. 2 and 3. Figure 2 shows an excellent coincidence with the frequencies of the mmWave and reasonable agreement with intensities of the mmWave modes with the lowest modes showing weak emission. Indeed, the fundamental mode at $\sim 26 \mathrm{GHz}$ is barely observed, although the second harmonic is weaker than the third, in contrast to the simulations. The detection of the fundamental electrical beatnote is discussed further below.

As well as simulating the expected spectrum, a power dependence measurement was performed to further show that the mechanism is based on a second-order nonlinearity. From the spectrum acquired from the temporal trace, the integral of the mmWave spectrum as a function of the integrated $\mathrm{THz}$ intensity was measured, with the $\mathrm{THz}$ intensity varied with the QCL bias (see Fig. 3). A power law is used to fit the experimental data and the best agreement is found for a value of the exponent $2.10 \pm$ 0.33 . This identifies the second-order nonlinear susceptibility, $\chi^{(2)}$, as the main contribution to the nonlinear optical process. The small deviation from an exponent equal to 2 is most likely a result of not efficiently detecting the low harmonics. To conclude, $\mathrm{QCL}_{1}$ shows equally spaced mmWave lines from 25 to $367 \mathrm{GHz}$ with an agreement between the mode intensity distribution of the measured and the calculated $\mathrm{GHz}$ lines through a second-order nonlinearity.

With the proof of principle of mmWave DFG demonstrated, $\mathrm{QCL}_{2}$, designed for ultra broadband $\mathrm{THz}$ emission, showed mmWave generation with an increased bandwidth extending to $500 \mathrm{GHz}$ (see Fig. 4a). However, owing to the irregular power distribution between the $\mathrm{THz}$ QCL Fabry-Pérot modes, the mmWave modal distribution is also irregular, in agreement with the simulations shown in Fig. 4b (blue curve), calculated as described above. Here, the expected spectrum is further compared to the case where the phases of the modes are assumed to be equal (black curve). Although the frequencies are the same, the modal intensities at low frequencies $(<100 \mathrm{GHz})$ are considerably higher than those measured. This highlights strongly the effect of the modal phases on the difference-frequency generation process in the case of neighbouring Fabry-Pérot modes. Furthermore, similar to observations in $\mathrm{QCL}_{1}, \mathrm{QCL}_{2}$ demonstrated that the 

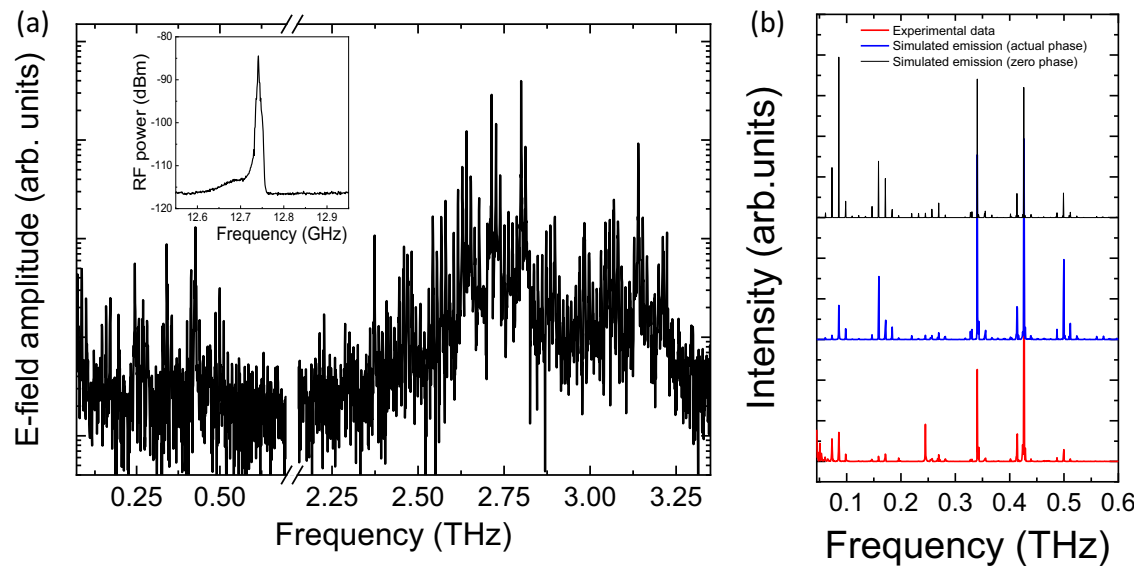

Fig. 4 mmWave generation from broadband $\mathbf{Q C L}\left(\mathbf{Q} \mathbf{C L}_{\mathbf{2}}\right)$. a Full amplitude spectrum showing $\mathrm{THz}$ emission centred at $2.8 \mathrm{THz}$ with a bandwidth of 800 $\mathrm{GHz}$ and nonlinear mmWave generation up to $500 \mathrm{GHz}$. Inset - fundamental electrical beatnote from THz QCL detected using the QCL as a detector and a spectrum analyser. $\mathbf{b}$ Enhanced view of mmWave intensity spectrum (red) compared to simulated emission for THz spectrum with measured phases (blue) and assuming equal phases (black). Spectra offset for clarity. The QCL is driven in pulsed mode (10\% duty cycle) and at a fixed heat sink temperature of $10 \mathrm{~K}$.

mmWave modes at low frequencies were weak compared to that expected, with the fundamental mode missing. However, as $\mathrm{QCL}_{2}$ was $3 \mathrm{~mm}$ long, the electrical and free-space fundamental beatnote is expected to be around $13 \mathrm{GHz}$. This was then electrically detected $^{36}$, using the QCL itself as an ultrafast detector and a standard spectral analyser (inset Fig. 4a), despite it being missing in the free space spectrum. This supports the above simulations for $\mathrm{QCL}_{1}$ that the free space optical modes at lower frequencies are not efficiently detected. This is a result of the strong diffraction owing to the subwavelength dimensions of the QCL waveguide, large focused spot sizes at these frequencies, combined with $\mathrm{THz}$ optics (parabolic mirrors) that have a limited collection efficiency. The detection of the narrow electrical beatnote also suggests a coherence between the Fabry-Pérot modes, which can be potentially locked to an external reference for modelocking. The high frequency microwave signals would also possess similar coherence properties.

For the final sample, $\mathrm{QCL}_{3}$, we show how the mmWave emission can be controlled to emit predominately at a single frequency by engineering the THz QCL spectral emission using dispersion management. Research using MIR QCLs ${ }^{30}$ has exploited imbedded gratings to force the QCL to emit at two distinct spectral lines for $\mathrm{THz}$ generation by DFG. Here we designed a THz QCL with an integrated $\mathrm{GTI}^{46}$ that permits the QCL to lase on distinct and separated spectral bands. Where previous work was based on placing the GTI off-resonance to reduce the total dispersion, here the centre of the QCL emission is placed in a dispersive regime that permits the generation of the spectral bands. The same active region as in $\mathrm{QCL}_{1}$ was used with a GTI of length $60 \mu \mathrm{m}$. The full emission spectrum of $\mathrm{QCL}_{3}$ is shown in Fig. 5a showing both the mmWave DFG and the THz emission. Figure $5 \mathrm{~b}$ shows an expanded view of the $\mathrm{THz}$ spectra, where the THz QCL emits in two $\mathrm{THz}$ spectral bands, each spanning several tens of gigahertz. The highest intensity modes are at $2.586 \mathrm{THz}$ and $2.342 \mathrm{THz}$ with a frequency separation of $0.244 \mathrm{THz}$. An expanded view of the mmWave spectra is shown in Fig. $5 c$ where the spectral position of the mmWave signal is in exact agreement with the difference in the frequencies of the main $\mathrm{THz}$ peaks. The intensity of the mmWave emission is equal to 0.02 percent of the highest pump intensity at $2.586 \mathrm{THz}$. This demonstrates the possibility of tailoring the desired mmWave spectrum by carefully engineering the $\mathrm{THz}$ emission, opening up the possibility of extending the emission towards $1 \mathrm{THz}$ using octave spanning QCLs. With $\mathrm{QCL}_{3}$ showing strong emission at mmWave frequencies, the time evolution of the spectrum was studied and shown in Fig. 6 as a colour plot. The intensities of the modes are plotted as a function of frequency $(x)$ and time in femtoseconds ( $y$-axis). This shows clearly that the mmWave emission (left curve) only takes hold after the QCL emission (right curve) is established and stable, here from $\sim 400$ ps. Figure 6 also shows the interesting dynamics of the system where before 400 ps corresponds to the initial transient stage where all modes experience some gain and hence amplified over a few round trips. Here it can be seen that the modes in-between the two main parts of the spectrum experience less gain owing to the GTI reflectivity. After 400 ps the laser reaches a steady-state region corresponding to laser action and where the cavity (GTI+laser) establishes the spectral emission. This behaviour is similar to the dynamics observed in DFB THz QCLs ${ }^{47}$, which was compared to a reduced rate formulism.

\section{Discussion}

Finally, the efficiency of the process can be used to determine the origin and the nonlinear susceptibility arising from differencefrequency generation ${ }^{25,30}$. The efficiency of $\mathrm{QCL}_{1}$, defined here as the ratio between the integrated mmWave and the $\mathrm{THz}$ spectrum, was found to be $\sim 1.7 \times 10^{-4}$. Previously work has indicated the possibility of phase matching in measurements of the microwave transmission $^{48}$ and pulse generation in $\mathrm{THz} \mathrm{QCLs}^{43,49}$. Here, using the time resolved nature of our experimental technique, we show directly the process is phase matched between the THz QCL emission and the nonlinear mmWave generation. Figure $7 \mathrm{a}$ shows the electric field of the $\mathrm{THz}$ QCL emission and the mmWave emission in the seeded regime. It is clear that the mmWave emission is in phase with the envelope of the $\mathrm{THz}$ emission, i.e., the phase velocity of the former is equal to the group velocity of the latter. This is a result of the low dispersion between these spectral ranges compared to the mid- and nearinfra-red and the relatively low mmWave losses in these microstrip-type waveguides. Taking this phase matched process, from the efficiency a second-order susceptibility $\chi^{(2)} \sim 1 \times 10^{5}$ $\mathrm{pm} / \mathrm{V}$ over the mmWave region is estimated with a $\mathrm{THz}$ intracavity power of $\sim 20 \mathrm{~mW}$. This giant nonlinearity when compared to GaAs $(\sim 100 \mathrm{pm} / \mathrm{V})$ is comparable to other intersubband systems and is a result of the large dipoles between closely spaced subbands $^{32}$. Indeed the calculated value from the QCL 

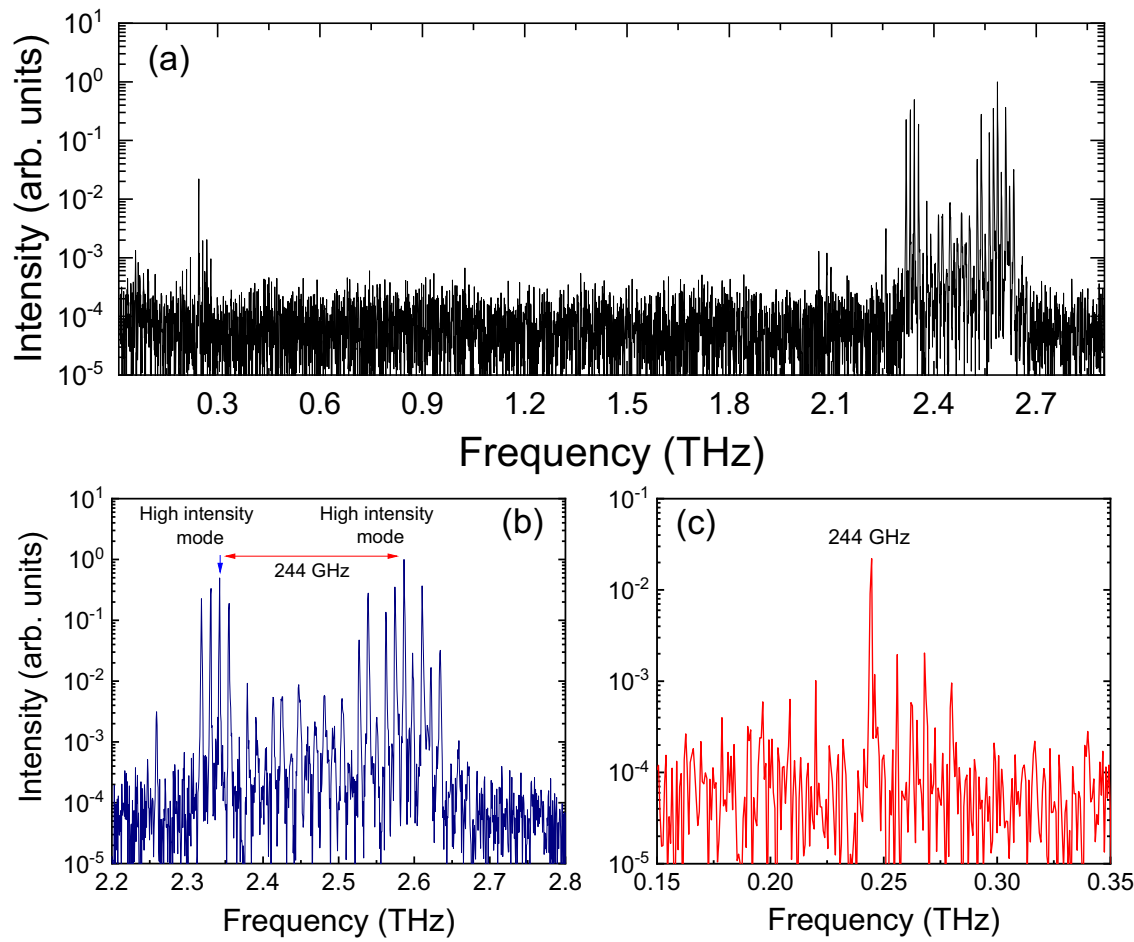

Fig. 5 mmWave emission engineering from $\mathbf{Q C L}_{\mathbf{3}}$ a Full spectrum showing $\mathrm{THz}$ emission split in two spectral bands around $2.45 \mathrm{THz}$ using an integrated GTI. Nonlinear mmWave generation centred at $244 \mathrm{GHz}$ is observed. $\mathbf{b}$ Enhanced view of $\mathrm{THz}$ emission showing the two spectral bands with mode separation of $14 \mathrm{GHz}$. c Enhanced view of nonlinear mmWave emission showing emission centred at $244 \mathrm{GHz}$. The QCL is driven in pulsed mode (10\% duty cycle) and at a fixed heat sink temperature of $10 \mathrm{~K}$.
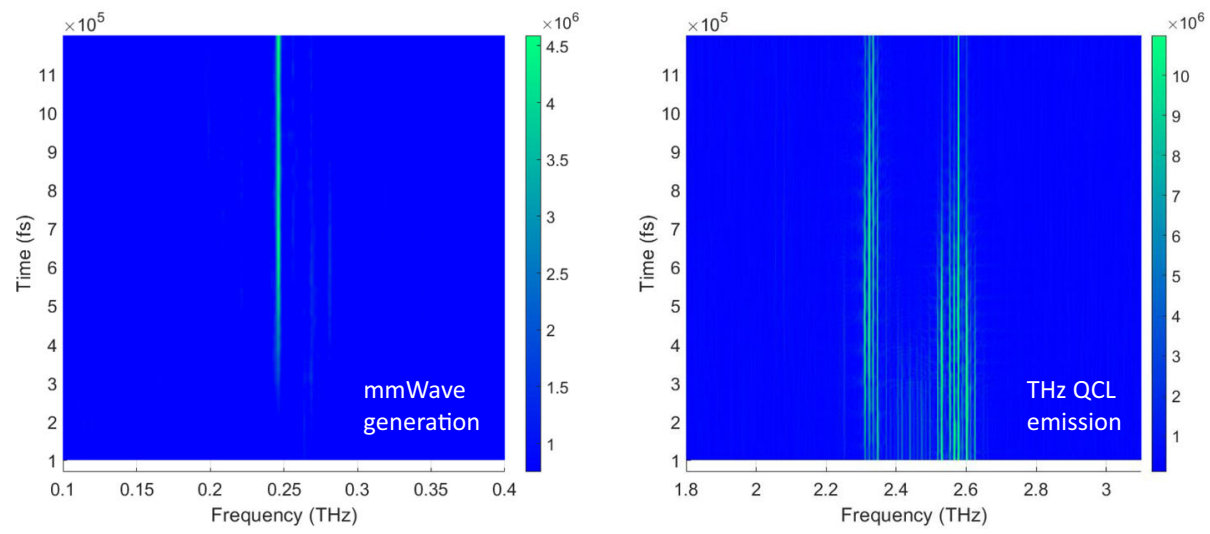

Fig. 6 Time resolved spectral emission from $\mathbf{Q C L}_{\mathbf{3}}$. The intensities of the spectral modes plotted as a function of frequency $(x)$ and time in femtoseconds (y-axis), showing the build-up and seeding of the $\mathrm{THz} \mathrm{QCL}$ emission at $\sim 400$ ps centred $\sim 2.45 \mathrm{THz}$ (right) followed by the generation of stable mmWave emission at $244 \mathrm{GHz}$ (left). The intensity of the emission (arb. units) is shown by the colour bar on the right of each figure.
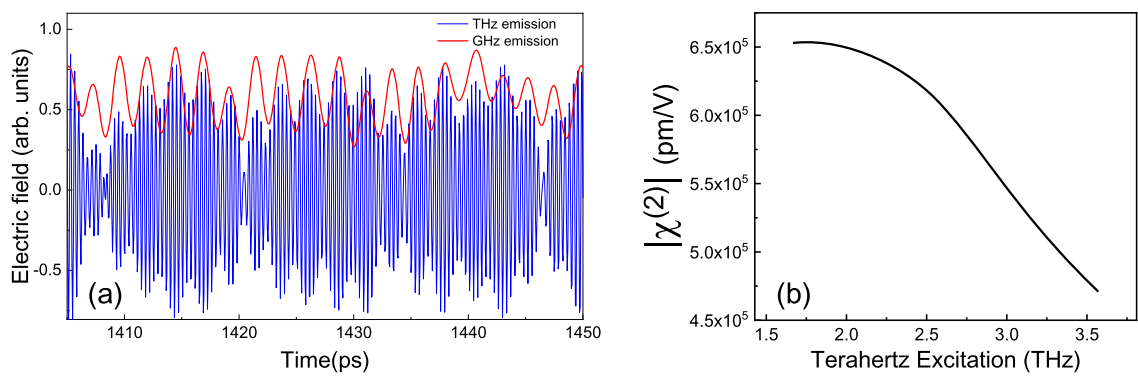

Fig. 7 Phase matching and nonlinear susceptibility between mmWave and THz emission. a Electric field as a function of time for both mmWave (red curve, filtered response of $\mathrm{THz}$ spectrum) and $\mathrm{THz}$ emission (blue) over a short time window illustrating the phase matched process between the two emission bands, b Modulus of second-order nonlinear susceptibility $\chi^{(2)}$ as a function of THz excitation energy, calculated from the QCL bandstructure, showing the exalted giant nonlinearity. 
bandstructure ${ }^{30}$ is $\chi^{(2)} \sim 6 \times 10^{5} \mathrm{pm} / \mathrm{V}$, highlighting that the nonlinearity is a result of resonant excitations of the subbands (see Fig. $7 \mathrm{~b}$ and supplementary material Fig. 4 for details on the simulation). The measured susceptibility is lower and could be a result of the lack of sensitivity of the detection scheme to low microwave frequencies as discussed above. Further, all the carrier population is assumed to be in the upper laser that tends to overestimate the nonlinearity. By engineering the dipole and nonlinear interferences between subbands ${ }^{50}$, further perspectives to engineer the response of the mmWave emission for higher efficiencies are possible.

To conclude, we demonstrate the generation of narrowband free space mmWave radiation that can cover the entire 26-500 $\mathrm{GHz}$ range. This is based on the beating of the Fabry-Pérot modes of THz QCLs and using electro-optic detection for sensitivity over the full microwave, mmWave and $\mathrm{THz}$ regions. A range of functionalities were shown with regular mode spacing up to 367 $\mathrm{GHz}$, mmWaves generated up to $500 \mathrm{GHz}$ using broadband QCLs and engineering of the mmWave emission by using integrated GTIs. The generation process was shown to rely on a second order process and thus indicates a second-order nonlinearity. The down-conversion of $\mathrm{THz}$ frequency lines into microwave and sub- $\mathrm{THz}$ emission is also a promising process to achieve continuous emission from a few $\mathrm{GHz}$ to several $\mathrm{THz}$ using multi-octave spanning QCLs. Further, owing to the demonstrated frequency comb nature of these sources and the increasing temperature operation of $\mathrm{THz}$ QCLs, the generation of extremely low-noise mmWave emission at hundreds of $\mathrm{GHz}$ is feasible. This would potentially permit the application of these sources as local oscillators for future high-frequency free space telecommunications.

\section{Methods \\ Experiment. The temporal characterisation of the $\mathrm{THz}$ quantum cascade laser (QCL) is based on coherent sampling of the electric field (E-field) using electro- optic detection. This technique requires the emission of the THz QCL to be phase locked to a THz pulse, and thus locked to the repetition rate of a femtosecond laser. An established ultrafast injection seeding technique is employed to realise this condition. A broadband $\mathrm{THz}$ seed pulse with a fixed phase is generated using an interdigitated photoconductive switch excited by a femtosecond Ti:Sapphire laser (see supplementary fig. 1) ${ }^{51}$. The THz pulse is injected into one end of the QCL cavity as an electrical radio frequency (RF) pulse (duration of a few nanoseconds), synchronised with the seed pulse, is used to gain switch the QCL. This permits the THz input pulse to be amplified, eventually seeding the QCL emission, avoiding laser action on the QCL's inherent spontaneous emission. The QCL emission is then measured using electro-optic sampling using a ZnTe crystal and a second beam from the same Ti:Sapphire laser.}

\section{Data availability}

The datasets generated and analysed during the current study are available in the zenodo repository, https://doi.org/10.5281/zenodo.4384637

Received: 2 November 2020; Accepted: 28 January 2021;

Published online: 03 March 2021

\section{References}

1. Nagatsuma, T., Ducournau, G. \& Renaud, C. C. Advances in terahertz communications accelerated by photonics. Nat. Photon. 10, 371-379 (2016).

2. Seeds, A. J. \& Williams, K. J. Microwave photonics. J. Light. Technol. 24, 4628-4641 (2006).

3. Capmany, J. \& Novak, D. Microwave photonics combines two worlds. Nat. Photon. 1, 319-330 (2007).

4. Wake, D., Lima, C. R. \& Davies, P. A. Optical generation of millimeter-wave signals for fiber-radio systems using a dual-mode DFB semiconductor laser. IEEE Trans. Microw. Theory Tech. 43, 2270-2276 (1995).

5. Eliyahu, D. \& Maleki, L. In IEEE MTT-S International Microwave Symposium Digest, 2003, Vol. 3. 2185-2187 (IEEE, 2003)
6. Novak, D., Ahmed, Z., Waterhouse, R. B. \& Tucker, R. S. Signal generation using pulsed semiconductor lasers for application in millimeter-wave wireless links. IEEE Trans. Microw. Theory Tech. 43, 2257-2262 (1995).

7. Fortier, T. M. et al. Generation of ultrastable microwaves via optical frequency division. Nat. Photon. 5, 425-429 (2011).

8. Li, J., Yi, X., Lee, H., Diddams, S. A. \& Vahala, K. J. Electro-optical frequency division and stable microwave synthesis. Science 345, 309 (2014).

9. Liu, J. et al. Photonic microwave generation in the X- and K-band using integrated soliton microcombs. Nat. Photon. https://doi.org/10.1038/s41566020-0617-x (2020).

10. Liang, W. et al. High spectral purity Kerr frequency comb radio frequency photonic oscillator. Nat. Commun. 6, 7957 (2015).

11. Rinaldi, N. \& Schröter, M. Silicon-Germanium Heterojunction Bipolar Transistors for mm-Wave Systems: Technology, Modeling and Circuit Applications (River Publishers, 2018).

12. Trew, R. J. High-frequency solid-state electronic devices. IEEE Trans. Electron Devices 52, 638-649 (2005).

13. Aghasi, H. et al. Terahertz electronics: application of wave propagation and nonlinear processes. Appl. Phys. Rev. 7, 021302 (2020).

14. Diddams, S. A., Vahala, K. \& Udem, T. Optical frequency combs: coherently uniting the electromagnetic spectrum. Science 369, eaay3676 (2020).

15. Köhler, R. et al. Terahertz semiconductor-heterostructure laser. Nature 417, 156-159 (2002).

16. Owschimikow, N. et al. Resonant second-order nonlinear optical processes in quantum cascade lasers. Phys. Rev. Lett. 90, 043902 (2003).

17. Dhillon, S. S. et al. The 2017 terahertz science and technology roadmap. $J$. Phys. Appl. Phys. 50, 043001 (2017).

18. Li, L. et al. Terahertz quantum cascade lasers with $>1 \mathrm{~W}$ output powers. Electron. Lett. 50, 309-311 (2014).

19. Turčinková, D. et al. Ultra-broadband heterogeneous quantum cascade laser emitting from 2.2 to $3.2 \mathrm{THz}$. Appl. Phys. Lett. 99, 191104 (2011).

20. Rösch, M., Scalari, G., Beck, M. \& Faist, J. Octave-spanning semiconductor laser. Nat. Photon. 9, 42-47 (2014).

21. Bosco, L. et al. Thermoelectrically cooled $\mathrm{THz}$ quantum cascade laser operating up to $210 \mathrm{~K}$. Appl. Phys. Lett. 115, 010601 (2019).

22. Kainz, M. A. et al. Thermoelectric-cooled terahertz quantum cascade lasers. Opt. Express 27, 20688-20693 (2019).

23. Khalatpour, A., Paulsen, A. K., Deimert, C., Wasilewski, Z. R. \& Hu, Q. Highpower portable terahertz laser systems. Nat. Photon. https://doi.org/10.1038/ s41566-020-00707-5 (2020).

24. Grange, T. et al. Room temperature operation of n-type Ge/SiGe terahertz quantum cascade lasers predicted by non-equilibrium Green's functions. Appl. Phys. Lett. 114, 111102 (2019).

25. Madéo, J. et al. All-optical wavelength shifting in a semiconductor laser using resonant nonlinearities. Nat. Photon. 6, 519-524 (2012).

26. Cavalié, $P$. et al. High order sideband generation in terahertz quantum cascade lasers. Appl. Phys. Lett. 102, 221101 (2013).

27. Li, L. H. et al. Broadband heterogeneous terahertz frequency quantum cascade laser. Electron. Lett. 54, 1229-1231 (2018).

28. Burghoff, D. et al. Terahertz laser frequency combs. Nat. Photon. 8, 462-467 (2014).

29. Garrasi, K. et al. High dynamic range, heterogeneous, terahertz quantum cascade lasers featuring thermally tunable frequency comb operation over a broad current range. ACS Photon. 6, 73-78 (2019).

30. Belkin, M. A. et al. Terahertz quantum-cascade-laser source based on intracavity difference-frequency generation. Nat. Photon. 1, 288-292 (2007).

31. Lu, Q. Y., Bandyopadhyay, N., Slivken, S., Bai, Y. \& Razeghi, M. Continuous operation of a monolithic semiconductor terahertz source at room temperature. Appl. Phys. Lett. 104, 221105 (2014).

32. Fujita, K., Hayashi, S., Ito, A., Hitaka, M. \& Dougakiuchi, T. Sub-terahertz and terahertz generation in long-wavelength quantum cascade lasers. Nanophotonics 8, 2235-2241 (2019).

33. Vijayraghavan, K. et al. Broadly tunable terahertz generation in mid-infrared quantum cascade lasers. Nat. Commun. 4, 2021 (2013).

34. Piccardo, M. et al. Radio frequency transmitter based on a laser frequency comb. Proc. Natl Acad. Sci. USA 116, 9181 (2019).

35. Piccardo, M. et al. Light and microwaves in laser frequency combs: an interplay of spatiotemporal phenomena. IEEE J. Sel. Top. Quant. Electron. 25, 1-12 (2019)

36. Gellie, P. et al. Injection-locking of terahertz quantum cascade lasers up to 35GHz using RF amplitude modulation. Opt. Express 18, 20799-20816 (2010).

37. Gallot, G., Zhang, J., McGowan, R. W., Jeon, T.-I. \& Grischkowsky, D. Measurements of the $\mathrm{THz}$ absorption and dispersion of $\mathrm{ZnTe}$ and their relevance to the electro-optic detection of THz radiation. Appl. Phys. Lett. 74, 3450-3452 (1999).

38. Oustinov, D. et al. Phase seeding of a terahertz quantum cascade laser. Nat. Commun. 1, 1-6 (2010). 
39. Freeman, J. R. et al. Laser-seeding dynamics with few-cycle pulses: MaxwellBloch finite-difference time-domain simulations of terahertz quantum cascade lasers. Phys. Rev. A 87, 063817 (2013).

40. Kumar, S., Williams, B. S., Kohen, S., Hu, Q. \& Reno, J. L. Continuous-wave operation of terahertz quantum-cascade lasers above liquid-nitrogen temperature. Appl. Phys. Lett. 84, 2494-2496 (2004).

41. Chassagneux, Y. et al. Low threshold $\mathrm{THz}$ QC lasers with thin core regions. Electron. Lett. 43, 41-42 (2007).

42. Fathololoumi, S. et al. Terahertz quantum cascade lasers operating up to $\sim 200$ $\mathrm{K}$ with optimized oscillator strength and improved injection tunneling. Opt. Express 20, 3866-3876 (2012).

43. Wang, F. et al. Generating ultrafast pulses of light from quantum cascade lasers. Optica 2, 944 (2015).

44. Wienold, M. et al. Low-voltage terahertz quantum-cascade lasers based on LO-phonon-assisted interminiband transitions. Electron. Lett. 45, 1030-1031 (1) (2009).

45. Mezzapesa, F. P. et al. Tunable and compact dispersion compensation of broadband $\mathrm{THz}$ quantum cascade laser frequency combs. Opt. Express 27, 20231-20240 (2019)

46. Wang, F. et al. Short terahertz pulse generation from a dispersion compensated modelocked semiconductor laser. Laser Photon. Rev. 11, 1700013 (2017).

47. Kundu, I. et al. Ultrafast switch-on dynamics of frequency-tuneable semiconductor lasers. Nat. Commun. 9, 3076 (2018).

48. Maineult, W. et al. Microwave modulation of terahertz quantum cascade lasers: a transmission-line approach. Appl. Phys. Lett. 96, 021108 (2010).

49. Wang, F. et al. Ultrafast response of harmonic modelocked $\mathrm{THz}$ lasers. Light Sci. Appl. 9, 51 (2020).

50. Houver, S. et al. Giant optical nonlinearity interferences in quantum structures. Sci. Adv. 5, eaaw7554 (2019).

51. Madeo, J. et al. Frequency tunable terahertz interdigitated photoconductive antennas. Electron. Lett. 46, 611-613 (2010).

\section{Acknowledgements}

The authors acknowledge funding from European Union under the Horizon 2020 research and innovation programmes FET-Open grant ULTRAQCL 665158, the European Research Council (ERC) consolidator grants SPRINT (681379) and LEON (820133), the French National Research Agency (ANR-18-CE24-0013-02 “TERASEL”), and EPSRC programme 'HyperTerahertz' (EP/P021859/1). E.H.L. acknowledges support from the Royal Society and the Wolfson Foundation.

\section{Author contributions}

V.P. and H.N. conceived and set up the experiment, acquired, and interpreted the experimental data. S.S.D. conceived the experimental concept. V.P. simulated the mmWave spectrum and S.H. developed the theoretical model for the nonlinearity. Sample growth was performed by L.L., E.H.L., and A.G.D., sample process was performed by P-B.V., R.C., K.G., and M.S.V. The manuscript was written and the data interpreted by V.P., H.N., A.G.D., M.S.V., and S.S.D.; J.M. and J.T. provided careful insights. All work was coordinated and overseen by S.S.D. All authors contributed to the discussion and to the final manuscript.

\section{Competing interests}

The authors declare no competing interests.

\section{Additional information}

Supplementary information The online version contains supplementary material available at https://doi.org/10.1038/s41467-021-21659-6.

Correspondence and requests for materials should be addressed to S.S.D.

Peer review information Nature Communications thanks Manfred Helm and the other anonymous reviewers for their contribution to the peer review of this work.

Reprints and permission information is available at http://www.nature.com/reprints

Publisher's note Springer Nature remains neutral with regard to jurisdictional claims in published maps and institutional affiliations.

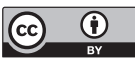

Open Access This article is licensed under a Creative Commons Attribution 4.0 International License, which permits use, sharing, adaptation, distribution and reproduction in any medium or format, as long as you give appropriate credit to the original author(s) and the source, provide a link to the Creative Commons license, and indicate if changes were made. The images or other third party material in this article are included in the article's Creative Commons license, unless indicated otherwise in a credit line to the material. If material is not included in the article's Creative Commons license and your intended use is not permitted by statutory regulation or exceeds the permitted use, you will need to obtain permission directly from the copyright holder. To view a copy of this license, visit http://creativecommons.org/ licenses/by/4.0/.

(c) The Author(s) 2021 